ORIGINAL ARTICLE-

Volume 12 Issue 32020

DOl: 10.21315/eimj2020.12.3.2

ARTICLE INFO

Submitted: $23-03-2020$

Accepted: 13-07-2020

Online: 30-09-2020

\section{Factors Influencing Career Choices Made by Internal Medicine Residents in Ahvaz, Iran}

\author{
Seyed Saeid Seyedian', Abdolhussein Shakurnia² \\ ${ }^{1}$ Internal Medicine Department, Alimentary Tract Research Center, \\ Imam Khomeini Hospital Clinical Research Development Unit, Ahvaz \\ Fundishapur University of Medical Sciences, Ahvaz, IRAN \\ ${ }^{2}$ Immunology Department, School of Medicine, Ahvaz Fundishapur \\ University of Medical Sciences, Ahvaz, IRAN
}

To cite this article: Seyedian SS, Shakurnia A. Factors influencing career choices made by internal medicine residents in Ahvaz, Iran. Education in Medicine Journal. 2020;12(3):11-19. https://doi. org/10.21315/eimj2020.12.3.2

To link to this article: https://doi.org/10.21315/eimj2020.12.3.2

\begin{abstract}
Little information is available on the factors affecting career choices of residents entering internal medicine (IM) specialty. So, this study was designed to assess the factors attractive for residents in choosing IM as a career choice. This cross-sectional questionnaire-based research was conducted in March 2019 at Ahvaz Jundishapur University of Medical Sciences, Iran. The questionnaire was distributed among all IM residents (75 residents). Responses were rated on a 5-point Likert scale. About 67 out of 75 questionnaires were completed and returned (response rate of $89.3 \%$ ). The criteria most often mentioned in the favour of choosing IM specialty were "improving knowledge and the treatment modality" (mean score: 4.25), "comprehensiveness of this specialty and the possibility of continuing education (fellowship)" (4.01), "personal interest" (3.89) and a "the need of community and serving people" (3.64). Regarding gender differences, "the need of community and serving people" $(p=0.001)$ and "high incomes" $(p=0.0001)$ were significantly more important for the choice of IM specialty by male residents. Our study provides a valuable insight into the factors that influence the career choice among IM residents. The findings from this study also suggest the need for a structured career-orientation programme at the institutional level that would guide the medical students in making their career choices.
\end{abstract}

Keyword: Internal medicine, Factors influencing, Specialty choice, Residents

CORRESPONDING AUTHOR Abdolhussein Shakurnia, Immunology Department, School of Medicine, Ahvaz Jundishapur University of Medical Sciences, Golestan St, Ahvaz, Iran | Email: shakurnia@yahoo.com

\section{INTRODUCTION}

Internal medicine (IM) is one of the most important specialties in the healthcare system that medical students can choose as a career. However, in recent studies reported that the number of medical students applying for residency in this field is declining. Recent studies conducted in
Iran, showed that IM specialty is no longer the most popular choice among medical students; and applying for this specialty is declining $(1,2)$. Obviously, if this trend continues, the decline in the number of IM graduates and the shortage of specialists in this field adversely affect the arrangement of health workforce in the community. Thus, there has been a rising concern about a 
decline in the number of medical students who wish to pursue a career in the field of IM.

The specialty choice decision by medical students has a direct impact on the supply of the workforce required in the healthcare system and contribute to the balance of the specialists required in each region. The imbalance in the health workforce is a major concern in the developed and developing countries (3). One of the challenges in health care is to match the number of newly graduated physicians to the future healthcare needs. Therefore, exploring on factors affecting medical students' decision on their specialty choices is crucial to achieve a balanced distribution of medical specialties.

Many studies demonstrated that the choice of medical practitioners for the specialty of their future careers are affected by many factors (3-5). These factors were classified in general, into intrinsic factors (e.g., for those related to personal attributes and preferences) and extrinsic factors (e.g., for those related to work environment). Some of these factors include income, controllable lifestyle, work-life balance, duration of training, number of on-calls, patient load and the flexibility of a specialty. Correspondingly, income and lifestyle have been found to be a critical factor influencing students' career choices. In addition, the personalities of medical students play a major role when choosing a particular specialty $(4,5)$.

Factors influencing specialty choice may somewhat vary in country to country and in different parts of the world. So, determining the factors influencing the specialty choice of IM residents is important to help the authorities of the medical school and health system planners by providing a realistic picture of residents' motivations in this regard. Career choice of medical students usually affect the distribution of physicians in different specialties across the country. To the best of authors' knowledge, in Iran, no study has been conducted on elucidating the contributing factors in the selection of IM as a career choice. Thus, a study to determine the factors that attract Iranian medical students towards IM as a future career is required. Without this information, an effective policy to attract medical residents to specialty suffering from insufficient manpower cannot be designed. Therefore, this study aimed to determine the factors that attract the choice of IM as a career in Ahvaz Jundishapur University of Medical sciences (AJUMS) in 2019.

\section{METHODS}

\section{Ethics Statement}

The study protocol was approved by the Ethics Committee of Institutional/ Regional Biomedical Research in AJUMS. All participants provided written informed consent. Questionnaires were anonymous and the residents were free to complete it.

\section{Study Design and Participants}

This cross-sectional study was conducted in March 2019 at the College of Medicine in AJUMS, Ahvaz, Iran. The sample size of the study included all 75 IM residents. The data collection tool was a valid and reliable questionnaire, which was used in previous studies $(1,2,6)$. This questionnaire was used after applying some changes in the current study. The validity and reliability of the instrument were reassessed and its reliability was confirmed by Cronbach's alpha coefficient of 0.792 .

\section{Survey Description}

An anonymous self-administered questionnaire was distributed to 75 medical residents, (from first to fourth academic years) recruited in the study. The questionnaire was consisted of two parts. The first part included demographic characteristics of the participants, while the second part was a five-point Likert questionnaire which had 16 items about 
reasons for choosing IM as a specialty. The instrument asked the participants to prioritise factors influencing their decision in choosing IM as their career by assigning a score from 1 (least important) to 5 (most important). The questionnaire was initially piloted over 20 residents for validation and the changes were introduced accordingly.

\section{Statistical Method}

The collected data were entered into the Microsoft Excel file and analysed using SPSS 16.0 version (Chicago, IL). In order to summarise residents' viewpoints and make better conclusion from the findings of the study, some of the questionnaire items were merged in terms of subject specificity. Finally, the questionnaire items called factors influencing the choice of IM were summarised in 10 items and analysed. Values were expressed as mean $\pm \mathrm{SD}$. The $t$-test was used to analyse the statistical differences between groups. The $p$-value of less than 0.05 was considered statistically significant.

\section{RESULTS}

The questionnaire was distributed among all the recruited internal residents. Out of the total 75 participating residents, 67 responded properly to the survey questionnaire with a response rate of $89.3 \%$.
Out of 67 residents $35(52.2 \%)$ were female and $40(59.7 \%)$ were married. The mean age of participants was $33.2 \pm 5.6$ years (females $31.6 \pm 1.3$ years and males 34.4 \pm 6.9 years). The majority of participants in the study were first- and second-year residents (61.2\%); and most of them $(60.3 \%)$ were accepted for their second and third choices. About two-thirds of the participants $(60.6 \%)$ had high satisfaction with their admission to IM course.

\section{Criteria for the Choice of IM Specialty}

The criteria most often mentioned in favour of choosing IM specialty were "improving knowledge and the treatment modality" (mean score: 4.25), "comprehensiveness of this specialty and the possibility of continuing education (fellowship)" (4.01), "personal interest" (3.89) and a "the need of community and serving people" (3.64). Moreover, "more convenience and lack of emergency" (2.36) and the "family and friend's advices" (2.83) were the least important criteria for the choice of IM specialty. Other criteria for the choice of IM specialty by the participants have been shown in Figure 1.

Regarding gender differences, "the need of community and serving people" ( $p=$ $0.001)$ and "high incomes" $(p=0.001)$ were significantly more important for the choice of IM specialty by male residents (Table 1). 


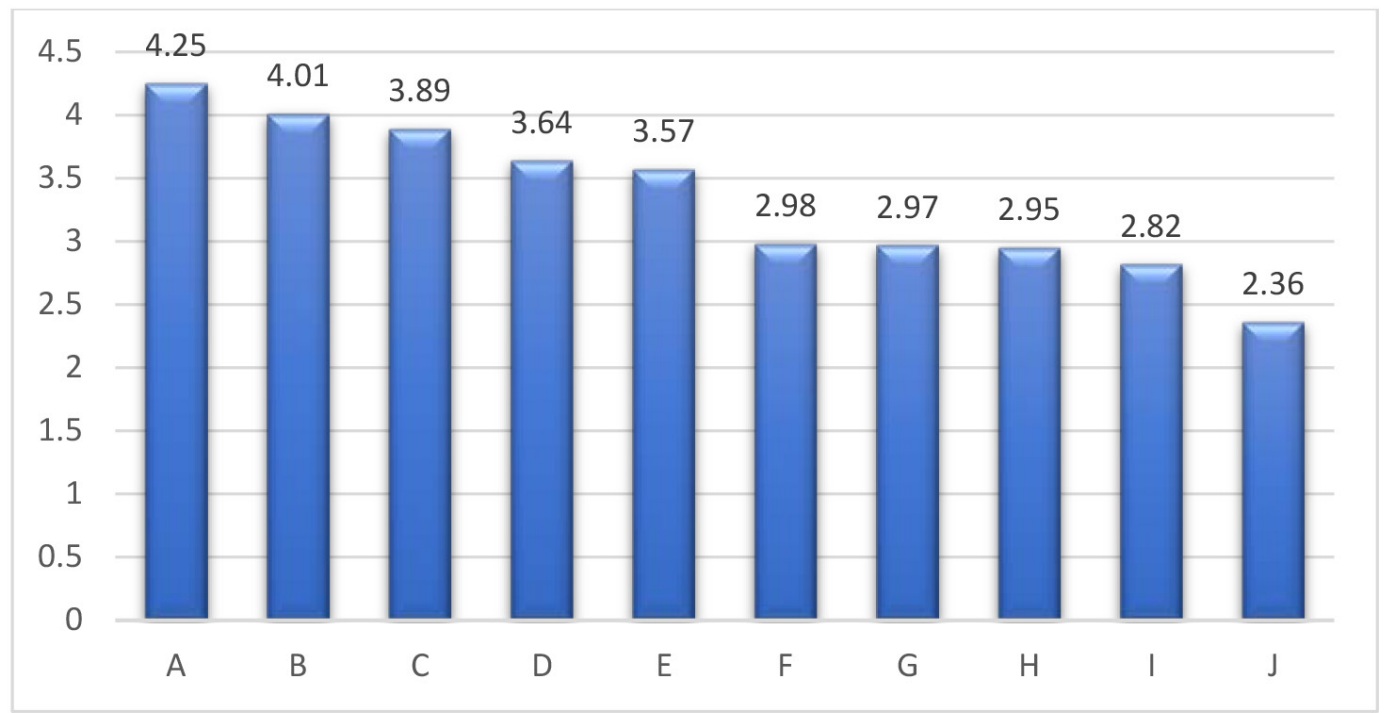

Figure 1: Factors affecting of the specialty choice in IM residents.

Notes: A: Improving knowledge and the treatment modality; B: Comprehensiveness of IM and the possibility of continuing education; C: Personal interest; D: The need of community and serving people; E: Good future career; F: Social prestige; G: High income; H: Not accepting in other specialised fields; I: Family and friend's advice; J: More convenience and lack of emergency.

Table 1: Student's $t$-test used to compare factors affecting of specialty choice among male and female IM residents

\begin{tabular}{|c|c|c|c|c|c|c|}
\hline \multirow[t]{2}{*}{ Factors } & \multicolumn{2}{|c|}{$\begin{array}{c}\text { Male } \\
(n=31)\end{array}$} & \multicolumn{2}{|c|}{$\begin{array}{l}\text { Female } \\
(n=34)\end{array}$} & \multirow[t]{2}{*}{$t$} & \multirow[t]{2}{*}{$p$-value } \\
\hline & Mean & SD & Mean & SD & & \\
\hline $\begin{array}{l}\text { Improving knowledge and the } \\
\text { treatment modality }\end{array}$ & 4.31 & 0.70 & 4.20 & 0.80 & 0.579 & 0.57 \\
\hline $\begin{array}{l}\text { Comprehensiveness of IM and the } \\
\text { possibility of continuing education }\end{array}$ & 4.18 & 0.93 & 3.86 & 0.90 & 1.41 & 0.16 \\
\hline Personal interest & 4.03 & 1.08 & 3.77 & 1.14 & 1.18 & 0.24 \\
\hline $\begin{array}{l}\text { The need of community and serving } \\
\text { people }\end{array}$ & 3.97 & 0.70 & 3.34 & 0.84 & 3.299 & 0.001 \\
\hline Good future career & 3.74 & 0.89 & 3.34 & 0.842 & 1.385 & 0.17 \\
\hline Social prestige & 3.16 & 0.86 & 2.82 & 0.87 & 1.57 & 0.12 \\
\hline High income & 3.35 & 0.98 & 2.63 & 0.70 & 3.43 & 0.001 \\
\hline $\begin{array}{l}\text { Not accepting in other specialised } \\
\text { fields }\end{array}$ & 3.06 & 0.93 & 2.86 & 1.03 & 0.983 & 0.33 \\
\hline Family and friend's advices & 3.05 & 0.97 & 2.61 & 0.94 & 1.844 & 0.07 \\
\hline $\begin{array}{l}\text { More convenience and lack of } \\
\text { emergency }\end{array}$ & 2.46 & 1.07 & 2.26 & 0.86 & 0.844 & 0.40 \\
\hline
\end{tabular}

Note: $p<0.05$ is significant. 


\section{DISCUSSION}

Although many studies are conducted on factors influencing the specialty choice of medical students, however, a few studies were specifically focused on IM residents. In the present study, many findings were particularly significant.

First, the improvement of personal knowledge, skills and competency to choose IM were more important than income; therefore, the factor "improving knowledge and treatment modality" was the most important in choosing IM and income had the least importance. This motive is rooted in a sacred and committed belief also reported in some other studies $(7,8)$. In a review study (4), the evaluation of 75 papers reported that getting professional qualifications was the second factor in the choice of specialty. This fact suggests that financial issues are not the important factor in the choice of IM specialty, a higher income cannot be the desired factor to prefer IM specialty and the problem of discouraging of IM residents cannot be solved though meeting this factor. Therefore, according to the findings of the present study, it seems that providing an appropriate educational environment to improve knowledge and skills of residents in clinical settings can be effective in improving their educational requirements and interest.

Second, the broadness of knowledge of IM and its multitude fellowship courses can greatly affect students' choice of specialty. In the present study, "comprehensiveness of IM and the possibility of continuing education (fellowship)" was the second most influential factor in the choice of IM, which could indicate the great interest of physicians in entering IM field for broader knowledge. The results of the studies showed that offering subspecialty and fellowship courses in IM increased the tendency of physicians towards choosing this specialty $(7,8)$. Hence, in order to meet this demand and raise the attention to IM specialty, administrators of medical education should increase the number of fellowship courses to attract the tendency of medical students towards this specialty.

Third, the interest is somewhat correlated with the two previous factors and expresses a profound scientific interest in the medical professionalism. The "personal interest", noted by many studies as one of the most important factors in the choice of specialty (9), was the third most influential factor in choosing IM specialty. The results showed that the previous interest to a specialty field will lead to increase medical students' interest during their studies in the medical school (10). Therefore, identifying the students' previous interests in IM and familiarising them with this field, and in particular its fellowship courses, can increase student interest and greater demand for IM specialty as a career.

It is appreciated and admired that the residents, after these three factors, noted the community needs and serving the people as the fourth factor in choosing IM specialty. This factor reflects the professional commitment of residents to the community and the people. The study on the motives of medical students in choosing IM specialty reported that the "social commitment" played a much more important role than "financial issues" (11). Newton and colleagues (12) showed that the medical residents also pay more attention to values in choosing specialty than money and social position. The attention to these values in specialty choice by medical students is very important. Besides, it can be as a main factor in responding to the society needs and the balance of health workforce in each region.

In the current study, like some other studies (13), IM residents less emphasised the role of factors such as "good future career", "social prestige", and "high incomes" in choosing specialty choice. However, in the present study, the factors lifestyle and income, which in some studies are the two important factors in choosing specialty (14, 15) were not among the first preferences 
of IM residents. However, the lack of attention to the social status and economic conditions of physicians in the community may have affected the unwillingness of medical students to IM specialty, which may cause problems in the future. Since the expectations of IM specialists from work and life conditions cannot be different from their counterparts living in other parts of the world, the role and importance of these factors in the choice of specialty should not be neglected. Therefore, the attention of policy makers in the health system to job satisfaction and livelihood status of IM specialists, as complementary factors, is important in the choice of this discipline.

The two factors, "convenience and lack of emergency" and "family and friend's advice", had the least importance in choosing IM specialty. This reflects the commitment and independence of residents to the choice of this specialty. It is a positive and worthwhile point that the IM residents prefer the community and people needs to their own convenience. In most studies, others' advice had the least role in choosing the specialty $(3,4,16)$, which was consistent with the findings of the current study and indicated the independence of residents in specialty choice. The finding of a study conducted in Sudan (9), showed the factors such as "avoid on-call" and "shorter work hours" were the least importance in choosing IM specialty as a career among undergraduate medical students, which is consistent with the findings of our study. Thus, it appears that Iranian medical students are like other medical students worldwide in their specialty choices.

In the current study, financial issues and high income were the important factors in choosing IM specialty by male residents. Since the male residents have more autonomy in life and mainly responsible for their own living expenditures and family life is also costlier for female residents, it is evident that income plays a more distinctive role in choosing a specialty by males. In the present study, "the need for community and serving people" was more important for male residents in choosing specialty. It was not in line with the results of a study showing that men were less inclined to choose the specialty to meet the community needs (3). The role of gender in career choices is well-studied and it was clarified that it is influenced by nationality and culture. In a qualitative study on Japanese female physicians regarding the specialty choice, it was observed that they mostly acted based on routine gender-based roles that focus more on family tasks and pay less attention to social and financial issues in specialty choice. It has been speculated that women seemed to have a more idealistic approach than men, and were less often influenced by the prospect of social prestige and good income (17).

Being aware of medical students' preferences in specialty choices and determine the influencing factors, it play a key role in determining the potential distribution of the specialist workforce in each area. This information is important in the planning of medical education, medical workforce and community health policies. By determining the factors influencing the specialty choice of residents and identifying their motivations in choosing the specialty, it can be improved the conditions for choosing specialty. Therefore, it can met the community needs to different specialties by justifying the students to choose the specialty based on their own interests and according to the community needs.

In conclusion, a variety of factors influence the residents of AJUMS when choosing IM as their preferred specialty. "Improving knowledge and treatment modality" and "comprehensiveness of IM and the possibility of continuing education (fellowship)" were considered the most attractive factors among IM residents; however, the least factors attracting were "more convenience and lack of emergency", as well as "family and friends" advices". 


\section{STRENGTHS AND LIMITATIONS}

This study had some limitations. First, the sample size is quite small although it is conducted at one of the biggest training university in Iran. Second, being a single centre study may not represent all the residents in Iran; so the results may not be generalised to other universities and countries. Further research in larger sample sizes in other universities and areas are required to evaluate and validate the factors influencing the choice of IM specialty. Despite the limitations, the present study conducted for the first time on IM residents at a great university in Iran and the results might be useful for the medical schools and health sector planners in the specialty choice process.

\section{FUTURE DIRECTION}

This study provides insights into the factors influencing specialty choice in Iranian IM residents. The results of the present study revealed the factors influencing the choice of IM by residents in Iran are slightly different from the ones found in the other countries. Being aware of identification students' viewpoints towards IM can be the basis to develop strategies to increase the attractiveness of IM specialty among medical students, since they choose their desired specialty according to these factors. The familiarity of medical students with IM specialty and its fellowships in educational classes and interactive programmes in clinical settings and hospitals leads to their hopeful interest. Further studies are required to identify and validate the factors influencing the choice of IM specialty at other universities and determine strategies to facilitate career choice in Iran. Undoubtedly, providing useful information helps health policymakers to appropriately plan and encourage medical students to choose IM specialty and improve the arrangement of medical workforce in the community. It is recommended further studies to be done across different medical universities in Iran for comparison and improving the educational programmes, resulting in developing a better healthcare system. The findings from this study also suggest the need for a structured careerorientation programme at the institutional level that would guide the medical students in making their career choices.

\section{CONCLUSION}

Understanding of the factors which encourage medical graduates towards IM for their future career or discourage them from it is required for policy making in specialist's manpower in this area. Determining why medical graduates choose their specialties is crucial in order to achieve a balanced distribution of clinicians in health care system. The proper understanding of the factors influencing physicians to choose IM specialty can help the policy makers to train human resources better based on the health system needs.

\section{ACKNOWLEDGEMENTS}

The authors wish to thank the IM residents for participating in the project and completed the questionnaire. The study protocol was approved by the Ethics Committee of Institutional/Regional Biomedical Research (ethical code: IR.AJUMS.REC.1397.914). All procedures performed in this study involving human participants were in accordance with the ethical standards. The main objective of the current research was explained to the students in the classroom and the verbal consent of participants was obtained. Questionnaires were anonymous and the students were free to complete it. Informed consent was obtained from all individual participants included in the study. 


\section{REFERENCES}

1. Alizadeh Y, Khoshbakht PM, Kazemnezhad LE, Khoshrang H, Behboudi H, Mohammadi $M$, et al. Factors associated with medical students tendency to choose a medical specialty. Journal of Guilan University of Medical Sciences. 2014;23(89):29-36.

2. Shakurnia A, Seyedian SS, Baniasad M. The tendency of senior medical students to choose a specialty: the difference between what students want and what society needs. Quarterly Educational Development of Jundishapur. 2019;10(3):209-18.

3. Grasreiner D, Dahmen U, Settmacher U. Specialty preferences and influencing factors: a repeated cross-sectional survey of first-to sixth-year medical students in Jena, Germany. BMC Med Educ. 2018;18(1):103. https://doi.org/10.1186/ s12909-018-1200-8

4. Yang Y, Li J, Wu X, Wang J, Li W, Zhu $\mathrm{Y}$, et al. Factors influencing subspecialty choice among medical students: a systematic review and meta-analysis. BMJ Open. 2019;9(3):e022097. https://doi.org/10.1136/ bmjopen-2018-022097

5. Alenezi $M$, Alharbi $H$, Alenezi $H$, Alharbi W, Alkhalaf B, Alsuhaibani R. Factors affecting future specialty choice in medical tnterns at Qassim University. Indo American Journal of Pharmaceutical Sciences. 2019;6(1):1382-9.

6. Momen A, Shakurnia A. Factors influencing pediatric specialty choice among pediatric residents of Ahvaz Jundishapur University of medical sciences. International Journal of Pediatrics. 2015;3(3.2):701-6.

7. Daniels VJ, Kassam N. Determinants of internal medicine residents' choice in the Canadian R4 fellowship match: a qualitative study. BMC Med Educ. 2011;11(1):44. https://doi.org/10.1186/1472-6920-11-44
8. Hauer KE, Durning SJ, Kernan WN, Fagan MJ, Mintz M, O'Sullivan PS, et al. Factors associated with medical students' career choices regarding internal medicine. JAMA. 2008;300(10):1154-64. https://doi. org/10.1001/jama.300.10.1154

9. Elzain YI, Alawad AAM, Khan WS, Khalil $\mathrm{HO}$, Abdelrazig YM, Ahmed OB, et al. Factors influencing the choice of internal medicine as a career among undergraduate medical students. International Journal of Health. 2014;2(2):22-5. https://doi. org/10.14419/ijh.v2i2.2918

10. Bonura EM, Lee ES, Ramsey K, Armstrong WS. Factors influencing internal medicine resident choice of infectious diseases or other specialties: a national cross-sectional study. Clin Infect Dis. 2016;63(2):155-63. https:// doi.org/10.1093/cid/ciw263

11. Correia LL, Mendonça VR, Garcia GB, Brandão EC, Barral-Netto M. Medical specialty choice and related factors of Brazilian medical students and recent doctors. PloS One. 2015;10(7):e0133585. https://doi.org/10.137 //journal. pone. 0133585

12. Newton DA, Grayson MS, Foster Thompson L. Money, lifestyle, or values? Why medical students choose subspecialty versus general pediatric careers. Clinical Pediatrics. 2010;49(2):116-22. https://doi. org/10.1177/0009922809350216

13. West CP, Drefahl MM, Popkave C, Kolars JC. Internal medicine resident self-report of factors associated with career decisions. J Gen Intern Med. 2009;24(8):946-9. https://doi.org/10.1007/s1 1606-009-1039-0

14. Khosravi H, Haitz K, Nambudiri VE. Residency specialty choice trends over 24 years. Journal of Medical Education. 2018;17(2):143-7. 
15. Horn L, Tzanetos K, Thorpe K, Straus SE. Factors associated with the subspecialty choices of internal medicine residents in Canada. BMC Med Educ. 2008;8(1):37. https://doi.org/10.1186/1472-6920-8-37

16. Qurat-ul-Ain K, Lodhi S, Abaidullah S. Factors influencing choice of internal medicine and subspecialties by postgrsduate residents in a tertiary care teaching hospital. Annals of King Edward Medical University. 2014;20(3):233-42.
17. Ie K, Murata A, Tahara M, Komiyama M, Ichikawa S, Takemura YC, et al. What determines medical students' career preference for general practice residency training? A multicenter survey in Japan. Asia Pac Fam Med. 2018;17(1):2. https://doi. org/10.1186/s12930-018-0039-9 